\title{
STATISTICAL ANALYSIS OF MECHANICAL DAMAGE IN ARRAYS OF MIXED NANOPILLARS
}

\author{
Tomasz Derda \\ Institute of Mathematics, Czestochowa University of Technology \\ Czestochowa, Poland \\ tomasz.derda@im.pcz.pl
}

\begin{abstract}
We apply the Fibre Bundle Model to study critical loads and catastrophic avalanches in arrays of axially loaded nanopillars under so-called local load sharing. Nanopillars with assigned random strength-thresholds are located in the nodes of the supporting square lattice. We analyzed different mixtures of weak and strong pillars, i.e. we use distributions of strength-thresholds drawn from two different uniform distributions.
\end{abstract}

Keywords: nanopillars, fibre bundle model, damage mechanics, statistics, skew normal distribution, periodic boundary conditions

\section{Introduction}

Failure of materials is a highly undesirable phenomenon whose dynamics attract much scientific attention, especially fractures and damages in heterogeneous materials. It is because of a broad area of technological applications of heterogeneous materials [1-3]. Due to complex relations between failures and subsequent stress transfer, the prediction of a sudden catastrophic failure is a serious problem for both the engineers and physicists. Several statistical models have been proposed to describe fracture processes in heterogeneous media. A very important class of these models are the Fibre Bundle Models (FBM) [3-5]. Despite their simplicity they capture the most important properties of material damage and breakdown. In the literature different realizations of the FBM [5-8] are proposed including the mixed models $[9,10]$.

In this paper we simulate the breakdown in arrays of weak and strong nanopillars assembled perpendicularly to a flat substrate [11]. Such arrays of nanopillars can be used as components in the systems of micromechanical sensors or in solar cells $[12,13]$. In [14] Ryu et al. have introduced model of fracture of silicon nanopillar arrays. Free-standing nanopillars can also be applied as elements in the micro- and nano-electromechanical systems, micro-actuators or optoelectronic devices $[15,16]$. The experimental tensile and compressive tests performed on 
single metallic micro- and nanopillars indicated significant strength increase via size reduction of the sample [15]. For this reason it is worth analysing mechanical destruction within an array of nano-sized pillars.

\section{Model of a mixed nanopillar array}

The system under consideration is composed of $N=L \times L$ longitudinal nanopillars located in the nodes of the square lattice of side length $L$. Each nanopillar is characterised by its own critical load $\sigma_{t h}^{i}$ to an applied axial load. In this sense $\sigma_{t h}^{i}$ is a strength-threshold of a given pillar. Pillar-strength-thresholds are uniformly distributed quenched random variables. It is assumed that the randomness of pillarstrength-thresholds $\sigma_{t h}^{i}, i=1,2, . ., N$ reflects the disorder of heterogeneous material. In the present study we employ a mixed scheme: part of the pillar-strengththresholds is drawn from the interval $(0,0.5)$ and the rest is randomized from the interval $(0.5,1)$. This ensures that each pillar with a strength-threshold drawn from the first interval is weaker than any pillar with a strength-threshold drawn from the second interval, therefore the first we call a weak pillar and the other - a strong pillar. We analyse five different arrangements (see Fig. 1):

- Graysystem - pillars are grouped in pairs of neighbouring elements. A weak pillar corresponds to 0 and a strong pillar corresponds to 1 in 2-bit Gray code,

- row system - rows of weak and strong pillars are arranged alternately,

- block system - weak and strong pillars are grouped in blocks $2 \times 2$,

- diagonal system - weak and strong pillars are arranged alternately on the diagonals,

- random system - weak and strong nanopillars are randomly placed (not included in Figure 1).
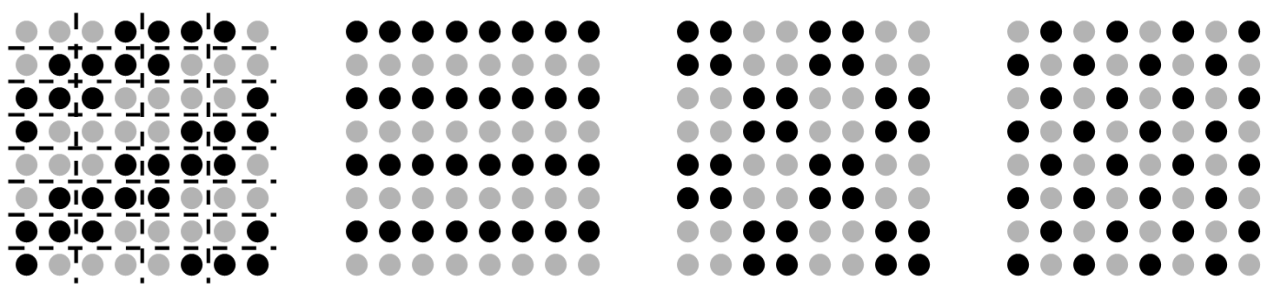

Fig. 1. Arrangements of weak (gray circles) and strong (black circles) nanopillars (from the left to the right): Gray system, row system, block system, diagonal system

The set of pillars is subjected to a quasi-statically increased external load $F$. At the beginning of the loading process all the nanopillars are intact and the external load $F=0$. The idea of quasi-static loading means that the external load is uniformly increased on all intact pillars until the destruction of one intact pillar under 
the load $\sigma_{i}$ exceeding strength-threshold $\sigma_{t h}^{i}$ of this pillar. After this destruction which is instantaneous and irreversible, the increase of the external load stops and the load coming from the damaged pillar is transferred to other intact pillars according to a given load transfer rule.

Several load transfer rules have been proposed, but the most commonly investigated are two extreme schemes: global load sharing (GLS) and local load sharing (LLS). The GLS rule represents the mean-field approach - the load coming from the destroyed pillar is equally redistributed to all intact pillars, therefore long-range interactions are assumed in the GLS rule. While, for the LLS rule only short-range interactions are observed - the load coming from the broken pillar is transferred to the nearest intact neighbours. Load transfer is not homogenous in that case and regions of stress concentration appear throughout the system. In the present paper we focus mainly on the LLS rule because for the GLS rule the arrangement of pillars is negligible. The results obtained for the GLS rule would be the same as for the systems with the pillar-strength-thresholds drawn uniformly from the interval $(0,1)$. In the following part of the work, the systems with pillar-strength-thresholds randomly chosen from the interval $(0,1)$ are called standard GLS and standard LLS, in dependence of applied load transfer rule.

Load redistribution increases stress on an intact pillar - it may cause further failures and then subsequent load transfers followed by possible failures. If the load transfer does not provoke failures a stable state appears. In this situation the external load $F$ has to be increased on the intact pillars in the same way as at the beginning of the loading process i.e. until damage of the weakest pillar. The loading process is continued until the whole nanopillar array collapses.

\section{Analysis of the simulation results}

We have performed computer simulations of the damage process in the quasistatically loaded mixed nanopillar arrays. In order to obtain reliable statistics, the calculations have been carried out for many samples. It should be noted that if it is not specified, we apply the LLS rule.

Damage process in the quasi-statically loaded nanopillar arrays proceeds as a sequence of simultaneous pillar crashes. This cascade of failures resembles sand or snow movement [17] and hence the number of destroyed pillars under an equal external load $F$ is called an avalanche and is denoted by $\Delta$. Each avalanche is initiated by an external load increase.

From the viewpoint of predicting a complete system breakdown two quantities are significant: critical load $F_{c}$ preceding complete failure and catastrophic (critical) avalanche $\Delta_{c}$ induced by exceeding of $F_{c}$. We analyse simulation results for these two quantities, which in fact are random variables. In order to compare results for different system sizes $F_{c}$ and $\Delta_{c}$ should be scaled by the appropriate initial system size $\sigma_{c}=F_{c} / N$ and $\Delta_{c} / N$. 


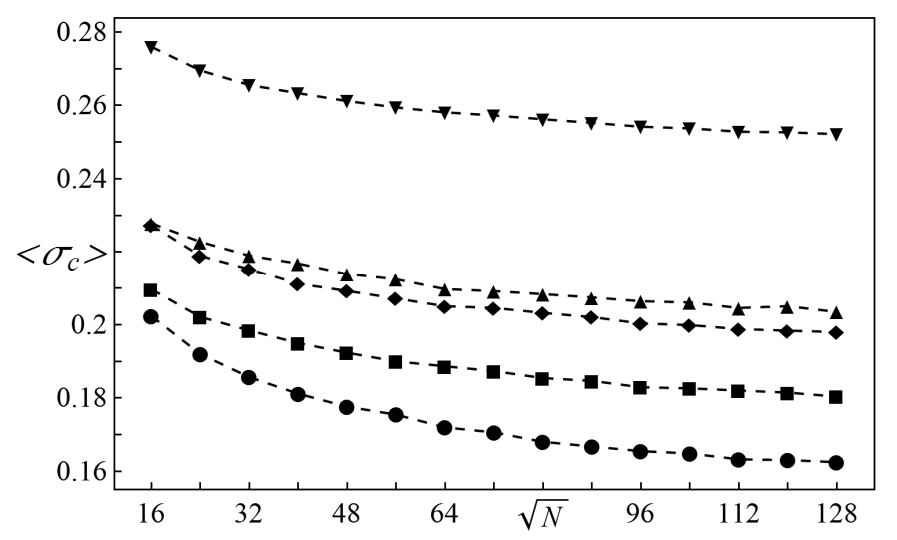

Fig. 2. The mean critical load $\sigma_{c}$ versus the linear array's size $L=\sqrt{N}$ for different configurations: random system (circles), Gray system (squares), row system (diamonds), block system (up triangles), diagonal system (down triangles). The averages are taken over at least 500 samples for each presented value

Figure 2 illustrates mean critical loads for different arrangements. It is seen that the arrangement of strong and weak pillars has a considerable influence on system strength. However with increasing system size the mean critical load $\left\langle\sigma_{c}\right\rangle$ decreases, but the ordering of $\left\langle\sigma_{c}\right\rangle$ for different system arrangements is preserved. This ordering can be explained by the neighbourhood rules. In the diagonal system each weak (strong) pillar has four strong (weak) nearest neighbours. In the row and block systems each strong (weak) pillar is surrounded by two strong and two weak nearest neighbours, but if the next-nearest neighbours are analysed, the differences appear (see Fig. 1). In the case of the Gray system two types of neighbourhood are visible - weak (strong) pillar is surrounded by a) two weak and two strong nearest neighbours, b) three weak (strong) and one strong (weak) nearest neighbours. All of the above discussed neighbourhoods do not concern boundary pillars.

The mean critical load for the standard GLS system asymptotically tends to 0.25 according to formula:

$$
\left\langle\sigma_{c}\right\rangle=0.25+0.30597 N^{-\frac{2}{3}}
$$

From Figure 2 it can be noticed that the diagonal system supports greater loads than the standard GLS system. The other analysed systems are definitely weaker having $\left\langle\sigma_{c}\right\rangle<0.23$.

The next investigated quantity is the catastrophic avalanche size. It is seen in Figure 3 that in the case of scaled sizes of catastrophic avalanche, the ordering visible in Figure 2 is reversed - the bigger the $\left\langle\sigma_{c}\right\rangle$, the smaller the critical avalanche. For the standard GLS systems, the scaled catastrophic avalanche asymptotically tends to 0.5 in accordance with: 


$$
\frac{\left\langle\Delta_{c}\right\rangle}{N}=0.5+0.4 N^{-\frac{2}{3}}
$$

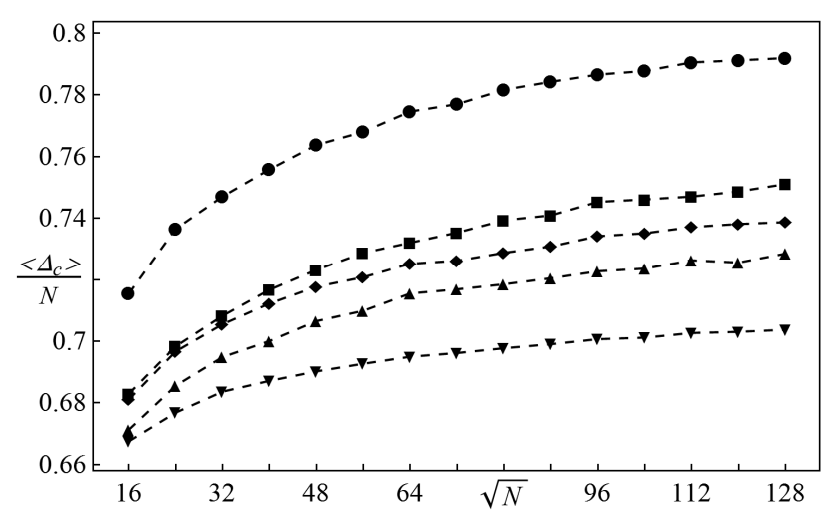

Fig. 3. The mean scaled size of the catastrophic avalanche vs. the linear array's size $L=\sqrt{N}$ for different configurations: random system (circles), Gray system (squares), row system (diamonds), block system (up triangles), diagonal system (down triangles).

The averages are taken over at least 500 samples for each presented value

In our analysed system $\left\langle\Delta_{c}\right\rangle / N>0.66$, meaning that the system will be completely destroyed if at most $1 / 3$ pillars in the array is broken.

In order to get a closer look at the results for the diagonal system we have performed simulations for system sizes $N>128 \times 128$. To avoid the boundary effects calculations have also been done for the diagonal systems with periodic boundary conditions (PBC). The results of $\left\langle\sigma_{c}\right\rangle$ and $\left\langle\Delta_{c}\right\rangle / N$ are graphically reported in Figures 4 and 5.

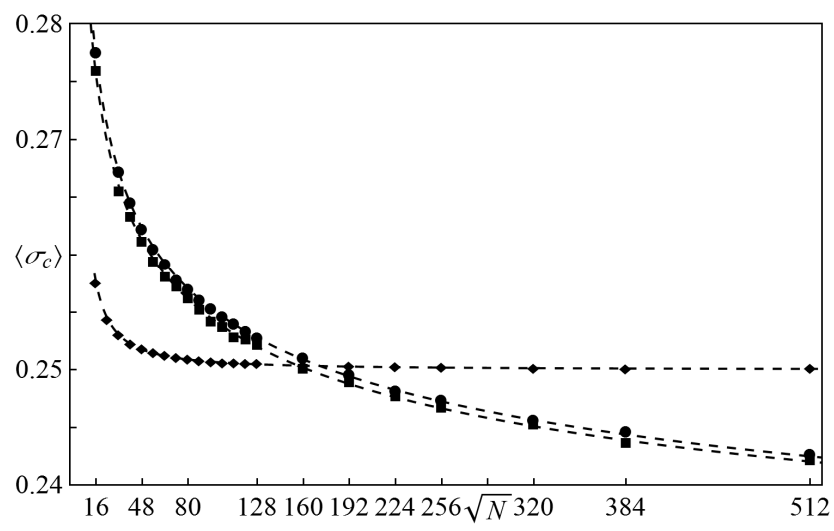

Fig. 4. The mean critical load $\sigma_{c}$ versus the linear array's size $L=\sqrt{N}$ for: diagonal systems without PBC (squares), diagonal systems with PBC (circles), standard GLS systems (diamonds) 


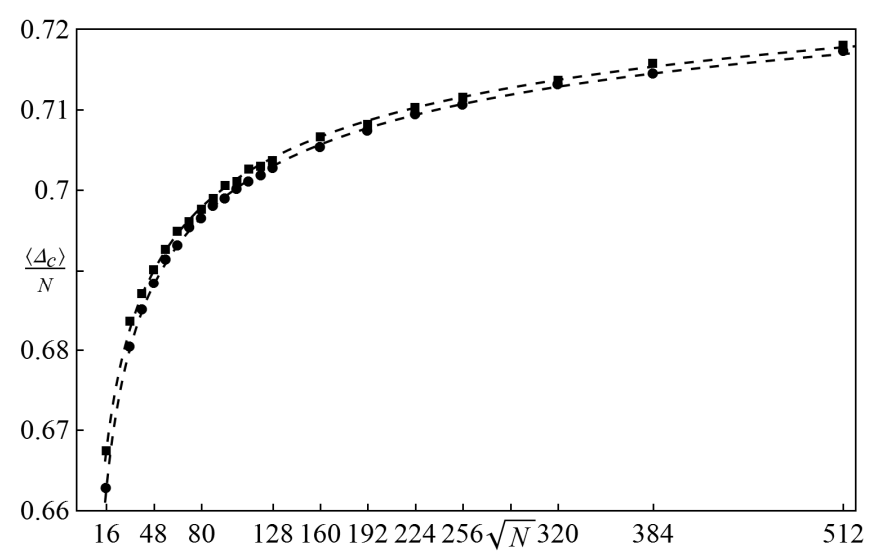

Fig. 5. The mean scaled size of the catastrophic avalanche vs. the linear array's size $L=\sqrt{N}$ for diagonal systems: without PBC (squares), with PBC(circles)

For the standard LLS system the mean values of $\sigma_{c}$ can be nicely fitted by the formula [18]:

$$
\left\langle\sigma_{c}(N)\right\rangle=\frac{1}{\lambda(\ln N)^{\delta}}
$$

with coefficients $\lambda=2.413$ and $\delta=0.414$. This formula is a good approximation for the diagonal system. Based on numerical results we have obtained $\lambda_{p b c}=2.721$, $\delta_{p b c}=0.165$ and $\lambda_{n p b c}=2.760, \delta_{n p b c}=0.160$ for the systems with and without PBC, respectively. Absolute percentage errors of these approximations are smaller than $0.14 \%$. For diagonal systems $\left\langle\sigma_{c}\right\rangle$ can also be approximated by the following equation:

$$
t(N)=\tau \frac{1}{\ln ^{2} N}+v \frac{1}{\ln N}+\xi
$$

with $\tau_{p b c}=-1.035, v_{p b c}=0.613, \xi_{p b c}=0.200$ and $\tau_{n p b c}=-1.022, v_{n p b c}=0.598$, $\xi_{n p b c}=0.201$. Absolute percentage errors of these approximations are smaller than $0.21 \%$. Figure 6 presents approximation errors for the diagonal system with PBC. It should be noticed that the relation (3) asymptotically tends to 0 , while $\lim _{n \rightarrow \infty} t(N)=0.2$ in the case of the above coefficient values.

As previously mentioned, the diagonal system is the only one analysed here for which $\left\langle\sigma_{c D L L S}\right\rangle>\left\langle\sigma_{c G L S}\right\rangle$ (see Fig. 4). However, function $\left\langle\sigma_{c D L L S}(N)\right\rangle$ decreases more quickly than $\left\langle\sigma_{c G L S}(N)\right\rangle$ and therefore for sufficiently large systems 
$\left\langle\sigma_{c G L S}\right\rangle>\left\langle\sigma_{c D L L S}\right\rangle$. We have found intersection points for these two curves $\left\langle\sigma_{c G L S}\right\rangle$ and $\left\langle\sigma_{c D L L S}\right\rangle: N \approx 155$ (without $\mathrm{PBC}$ ), $N \approx 170$ (with PBC).

It is interesting that formula (4) can be used as a scaling relation also for $\left\langle\Delta_{c}\right\rangle / N[18,19]$ where $\tau_{p b c}=0.894, v_{p b c}=-0.771, \xi_{p b c}=0.773$ and $\tau_{n p b c}=1.286$, $v_{n p b c}=-0.834, \xi_{n p b c}=0.776$ for diagonal systems with PBC and without PBC, respectively. Absolute percentage errors of these approximations do not exceed $0.12 \%$.

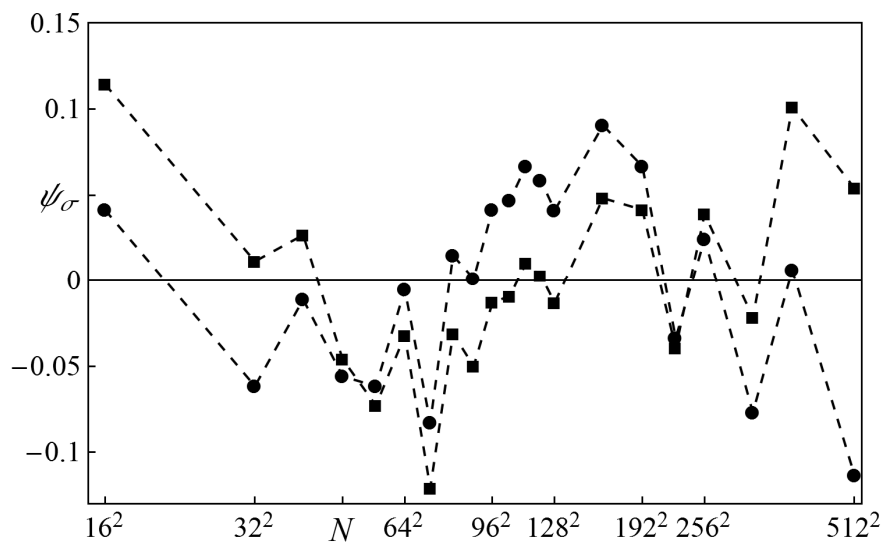

Fig. 6. Percentage errors of $\left\langle\sigma_{c}\right\rangle$ approximation using: formula (3) - squares, formula (4) - circles. The results concern diagonal systems with PBC

In the last part of the chapter we study the distribution of critical load values and catastrophic avalanche sizes for diagonal systems with PBC. Exemplary empirical probability density functions of these two quantities have been shown in Figures 7 and 9. Critical load $\sigma_{c}$ is a continuous random variable which follows three-parameter skew normal distribution (see Fig. 7) being a generalization of Gaussian distribution for non-zero skewness [20]. The distribution of $\sigma_{c}$ is characterised by negative skewness for all analysed system sizes (from $N=16^{2}$ to $N=512^{2}$ ).

Concerning prediction of a system failure, it is important to know the probability of the system destruction under a given load. In that situation it is convenient to work with the complement of the cumulative distribution function namely the survival function $\operatorname{sf}\left(\sigma_{c}\right)=1-\operatorname{cdf}\left(\sigma_{c}\right)$. This function gives a probability that the system is working under load $\sigma_{c}$. Chosen empirical survival functions are illustrated in Figure 8 and it is seen that the bigger the system, the smaller the probability that it supports a given load $\sigma_{c}$. 


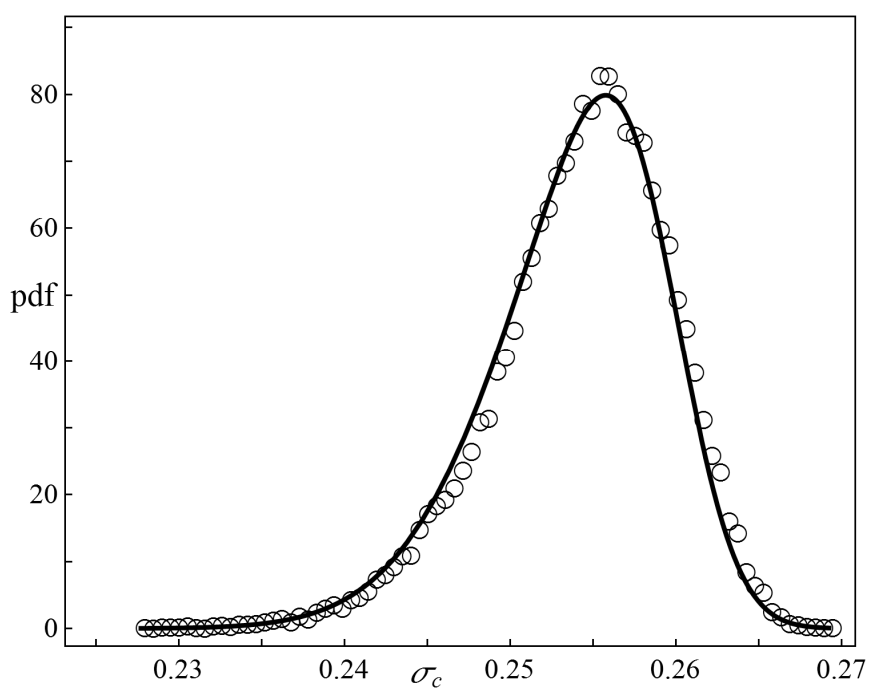

Fig. 7. Probability density function of the $\sigma_{c}$ in an array with $112 \times 112$ pillars obtained from 30000 samples. Here the results for the diagonal system with PBC are shown and the solid line represents skew-normally distributed $\sigma_{c}$ with parameters computed from these samples

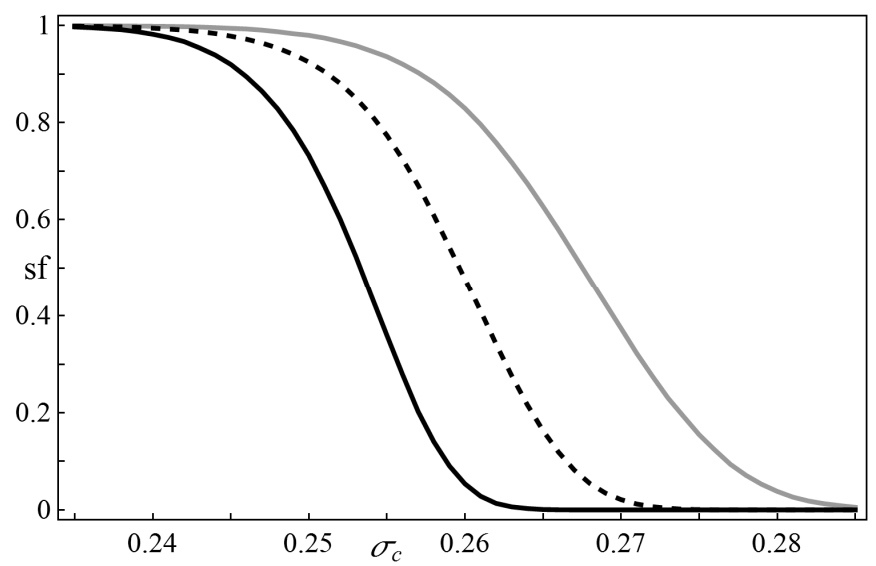

Fig. 8. The empirical survival functions (sf) of critical load $\sigma_{c}$ in an arrays of $32 \times 32$ (grey line), $64 \times 64$ (dashed line), $128 \times 128$ (black line) nanopillars. Each survival function was built on at least 30000 independent configurations of diagonal system with PBC

Although critical avalanche size $\Delta_{c}$ is a discrete random variable and skew normal distribution is a continuous probability distribution, we have approximated $\Delta_{c}$ by this distribution (see Fig. 9). We have performed Cramer-von Mises and Anderson-Darling tests. The hypothesis that $\Delta_{c}$ follows a skew normal distribution was not rejected at the $5 \%$ significance level for systems with $N \geq 64^{2}$ pillars. The 
hypothesis was rejected for smaller system sizes, namely $N \in\left\{24^{2}, 32^{2}, 48^{2}\right\}$. The distribution of $\Delta_{c}$ is characterised by positive skewness for systems with sizes $N \geq 24^{2}$ pillars. To gain a more complete insight into the distribution of $\Delta_{c}$ the results for different system sizes have been shown in Figure 10.

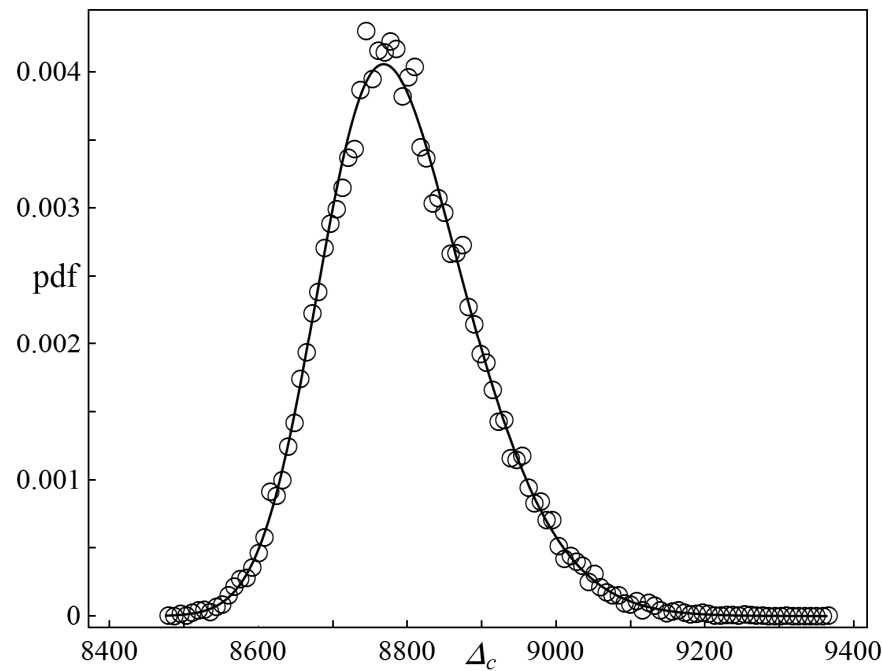

Fig. 9. Probability density function of the $\Delta_{c}$ in an array with $112 \times 112$ pillars obtained from 30000 samples. Here the results for the diagonal system with PBC are shown and the solid line represents skew-normally distributed $\Delta_{c}$ with parameters computed from these samples

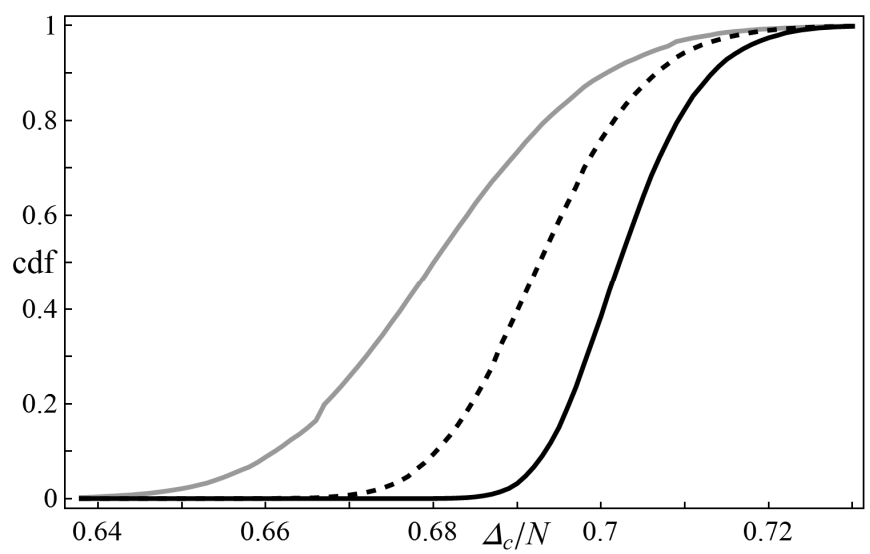

Fig. 10. The empirical cumulative distribution functions of the scaled critical avalanche $\Delta_{c} / N$ in an arrays of $32 \times 32$ (grey line), $64 \times 64$ (dashed line), $128 \times 128$ (black line) nanopillars. Each cdf was built on at least 30000 independent configurations of a diagonal system with PBC 


\section{Conclusion}

We have studied critical loads and catastrophic avalanches in arrays of weak and strong nanopillars. The pillars were quasi-statically loaded and we applied a local load sharing rule after each pillar breakdown.

Based on simulation results, we have noticed ordering of critical loads and catastrophic avalanche sizes according to an arrangement of weak and strong pillars. The strongest analysed system is the diagonal one. For this system we have fitted mean critical loads and mean catastrophic avalanche size by formulas (3) and (4). It has been shown that critical load and catastrophic avalanche size distributions are well approximated by skew normal distributions.

\section{References}

[1] Herrmann H.J., Roux S. (eds.), Statistical Models for the Fracture of Disordered Media, North Holland, Amsterdam 1990 and references therein.

[2] Chakrabarti B., Benguigui L.G., Statistical Physics of Fracture and Breakdown in Disordered Systems, Clarendon Press, Oxford 1997.

[3] Alava M.J., Nukala P.K.V.V., Zapperi S., Statistical models of fracture, Adv. in Physics 2006, 55, 349-476.

[4] Pradhan S., Hansen A., Chakrabarti B.K., Failure processes in elastic fiber bundles, Rev. Mod. Phys. 2010, 82, 499-555.

[5] Hidalgo R.C., Moreno Y., Kun F., Herrmann H.J., Fracture model with variable range of interaction, Phys. Rev. E 2002, 65, 046148.

[6] Hidalgo R.C., Zapperi S., Herrmann H.J., Discrete fracture model with anisotropic load sharing, J. Stat. Mech. 2008, P01004.

[7] Hidalgo R.C., Kovacs K., Pagonabarraga I., Kun F., Universality class of fiber bundles with strong heterogeneities, EPL 2008, 81, 54005.

[8] Lehmann J., Bernasconi J., Breakdown of fiber bundles with stochastic load-redistribution, Chemical Physics 2010, 375(2-3), 591-599.

[9] Divakaran U., Dutta A., Critical behavior of random fibers with mixed Weibull distribution, Phys. Rev. E 2007, 75, 011109.

[10] Divakaran U., Dutta A., Random fiber bundle with many discontinuities in the threshold distribution, Phys. Rev. E 2008, 78, 021118.

[11] Chekurov N., Grigoras K., Peltonen A., Franssila S., Tittonen I., The fabrication of silicon nanostructures by local gallium implantation and cryogenic deep reactive etching, Nanotechnology 2009, 20, 65307. Also: http://nanotechweb.org/cws/article/tech/37573

[12] Mariani G., Zhou Z., Scofield A., Huffaker D.L., Direct-bandgap epitaxial coremultishell nanopillar photovoltaics featuring subwavelength optical concentrators, Nano Lett. 2013, 13(4), 1632-1637.

[13] Mariani G., Scofield A., Hung C.-H., Huffaker D.L., GaAs nanopillar-array solar cells employing in situ surface passivation, Nat. Commun. 2013, 4, 1497.

[14] Ryu I., Lee S.W., Gao H., Cui Y., Nix W.D., Microscopic model for fracture of crystalline Si nanopillars during lithiation, Journal of Power Sources 2014, 255, 274-282.

[15] Greer J.R., Jang D., Kim J.-Y., Burek M.J., Emergence of new mechanical functionality in materials via size reduction, Adv. Functional Materials 2009, 19, 2880-2886. 
[16] Chekurov N., Fabrication process development for silicon micro and nanosystems, $\mathrm{PhD}$ thesis, Aalto University, Helsinki 2011. Available: http://lib.tkk.fi/Diss/2011/isbn9789526035932/

[17] Reiweger I., Schweizer J., Dual J., Herrmann H.J., Modeling snow failure with a fibre bundle model, Journal of Glaciology 2009, 55, 997-1002.

[18] Domański Z., Derda T., Sczygiol N., Critical avalanches in fiber bundle models of arrays of nanopillars, Proceedings of the International MultiConference of Engineers and Computer Scientists 2013, Vol II, IMECS 2013, March 13-15, 2013, Hong Kong.

[19] Domański Z., Derda T., Sczygiol N., Statistics of critical avalanches in vertical nanopillar arrays, Lecture Notes in Electrical Engineering 2014, 275, 1-11.

[20] Azzalini A., A class of distributions which includes the normal ones, Scand. J. Statist. 1985, 12, 171-178. 